\title{
Application of Geometry Rectification to Deformed Characters Recognition
}

\author{
Liqun Wang, ${ }^{1}{ }^{*}$ and Honghui Fan ${ }^{2}$ \\ ${ }^{1}$ School of Information Engineering, Northeast Dianli University, Jilin, Jilin, 132012, China \\ ${ }^{2}$ Computer Engineering School, Jiangsu University of Technology, Changzhou Jiangsu, 213001, \\ China \\ aguoshuqiang@gamil.com
}

Keywords: Deformed characters; Character recognition; Geometry rectification; Machine vision

\begin{abstract}
Due to the complexity of text deformation, it brings great difficulties to the character recognition. In this paper, we propose an algorithm based on geometry rectification to identify the deformed characters, which improves the accuracy and recall rate of text recognition. In this method, the deformed character is rectified within a circle using the moments of deformed character, and then it can be recognized in real time by comparing the similarity of distance histogram and angle histogram. Experimental results show that the method based on affine invariant can recognize the English characters and numbers with a very high recognition rate.
\end{abstract}

\section{Introduction}

In recent years, with the development of digital camera manufacturing technology, the quality of the digital camera has been greatly enhanced. Ten megapixels of the digital camera's max resolution has become mainstream configuration, and megapixel of the mobile phone camera has also appeared in the market. In addition to the increasing performance of the digital camera in hardware, the software is also continuous improvement. Now, the user can easily get a clear, professional digital image by means of image processing technology such as automatic stitching, face recognition, image stabilizer and so on. Under this background, there has been increasing attention to the character recognition technology in the digital image. In the character recognition technology the image, researchers carried out research into the character recognition in the field of natural scene understanding, vehicle license plate recognition, digital image libraries, geographic information system and so on, and so many mature commercial software also appeared. In recent years, a smartphones translation application based on character recognition has been developed by NetEase Co.Ltd. This application can translate the English word to Chinese word in real-time using the extract English word from the picture caught by smartphone camera. Because it can eliminate the process of inputting words manually, so many people use it benefited from its speediness and convenience.

However, there are so many problems must be solved in the field of character recognition in the image caught by camera. Among them, deformation generated by the angle between the focal plane of camera and text plane is the main cause of character recognition error. The English word rotates along the $\mathrm{x}, \mathrm{y}$ and $\mathrm{z}$-axis respectively. According to results, when the rotation angle along the $\mathrm{x}$, $\mathrm{y}$-axis is more than 36-degree, the correct recognition rate decreases dramatically. Similarly, when the rotation angle along the along the $\mathrm{z}$-axis is more than 13-degree, the correct recognition rate decreases dramatically. Therefore, the recognition robustness to rotation along all directions is necessary for user. At the same time, the character recognition algorithms in smartphones, digital cameras and other embedded systems must not damage the real-time performance of the device.

At present, many scholars have carried out the researches on character recognition of deformed characters [1-2]. M. Ryan and C. Liu pick up the whole line in image, according to the gradient of the whole line the character is corrected and then is recognized [3-5]. This method is very effective to the case that the whole line can be extracted. However, if the whole line feature is not obvious in the image, the recognition rate would decrease remarkably. J. I. Olszewska presents a method that the characters are equivalent to the approximate ellipse [6-8]. The approximate ellipse is corrected to a 
perfect circle, and then the character is recognized in the circle. This method is very effective to the deformation caused by perspective. However, it is not available to the deformation caused by rotation. In order to recognize the deformation character caused by rotation, P. M. Patil compares the sample character with the rotating standardization character, and then recognizes the text [9-10]. This method must rotate the character continually, so it destroys the real-time requirement of character recognition inevitably.

In this paper, a novel high speed character recognition method is proposed. In this method, the deformed character is corrected by the affine invariance, and then the distance histogram and angle histogram of corrected character is compared with the standard sample character. The experiment result shows that the method proposed in this paper can recognize the rotated word along all directions in real-time.

\section{Character Recognition Method Based on Geometry Correction}

The process of character recognition using geometry correction is as shown in Fig. 1. Firstly, the reformatted character is standardized by geometry correction. Secondly, the distance histogram and angle histogram of standardization character is compared with standard sample character to recognize the character. Finally, according to the comprehensive index, the character recognition results are output.

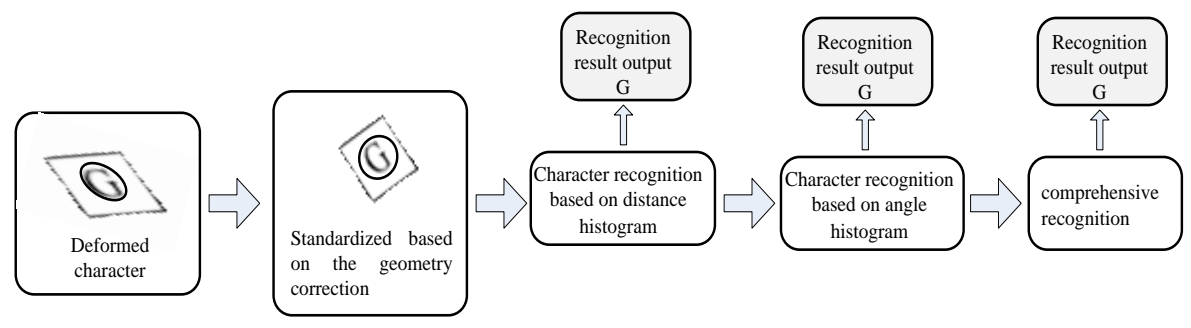

Figure 1. Character recognition process

As shown in Fig. 1, the deformed character is included in an approximate ellipse firstly, and then approximate ellipse is corrected into a standard circle. Because the deformed character and the approximate ellipse have the same affine property, when the approximate ellipse is corrected into a standard circle, the deformed character is correct into a standard character.

The approximate ellipse of deformed characters is determined by the covariance matrix, and the covariance matrix calculated by:

$$
C=\left[\begin{array}{ll}
\frac{m_{20}}{m_{00}} & \frac{m_{11}}{m_{00}} \\
\frac{m_{11}}{m_{00}} & \frac{m_{02}}{m_{00}}
\end{array}\right]
$$

Where $\mathrm{m} 00, \mathrm{~m} 11, \mathrm{~m} 02, \mathrm{~m} 20$ are the zeroth order moments and second order moments. The calculation equation is given as:

$$
\begin{aligned}
& m_{i j}=\iint x_{i} y_{j} f(x, y) d x d y \\
& f(x, y)= \begin{cases}1 & (x, y) \in \text { character region } \\
0 & (x, y) \in \text { non-character region }\end{cases}
\end{aligned}
$$

The deformed character is corrected into a standard character by the inverse matrix $\mathrm{C}-1$ of covariance matrix in the image. We assume that the coordinates of deformed character image is (x,y) and the coordinates of corrected character image is $\left(\mathrm{x}^{\prime}, \mathrm{y}^{\prime}\right)$. Then relationships between the original coordinate and corrected coordinate is given as:

$$
\left[\begin{array}{l}
x^{\prime} \\
y^{\prime}
\end{array}\right]=C^{-1}\left[\begin{array}{l}
x-m_{10} \\
y-m_{01}
\end{array}\right]+\left[\begin{array}{l}
m_{10} \\
m_{01}
\end{array}\right]
$$

$m_{10}, m_{01}$ is the first order moment, its calculating methods as shown in Equation 2. 
As shown in Fig. 1, the rotation angle still exists in the standardized character image probably, so standardized character cannot be compared with the sample characters immediately. The rotation of character must be considered for recognition.

In traditional method, the deformed character is rotated at a certain angle. The rotated character is compared with sample character, and the recognition result is output according to the similarity. The correction recognition rate of this method is closely related to the rotation angle interval. When the interval angle is small, the correction recognition rate will be greatly increased. However, it can't satisfy the real-time requirement of character recognition in embedded device due to the increase of the amount calculation.

As shown in Fig. 1, a novel recognition method is proposed in this paper on the basis of combining distance histogram and angle histogram recognition method. This method is able to improve the recognition accuracy. At the same time, it can also guarantee real-time feature of embedded device.

The all character pixel whose distance is equidistant from the pixel to the gravity center of character is called distance histogram. The radius of standard circle Standardized treated by geometry correction is R. We assume that the number of bin in standard circle is $\mathrm{n}$, and the total number of bin is $\mathrm{k}$. Then the relationship between $\mathrm{n}$ and $\mathrm{k}$ is given as:

$$
n= \begin{cases}k-1 & k<10 \\ k-2 & k \geq 10\end{cases}
$$

In another word, the standard circle is divided into $\mathrm{n}$ shares along radial direction, and the radius difference between two concentric circles is $\mathrm{R} / \mathrm{n}$. Because there are character pixels outside the standard circle, one or two circles are added outside the standard circle according to the value $\mathrm{k}$. The distance histogram can be draw by counting the number of character pixels between adjacent two concentric circles. Distance histogram can be normalized by adjusting the total number of distance histogram bins into 1 .

Normalized distance histogram is regarded as an $\mathrm{n}$ dimension vector, and the number of pixels in each bin is regarded as a vector. The similarity between image character and sample character is calculated as:

$$
\rho(X, Y)=\frac{\operatorname{cov}(X, Y)}{\sigma_{X} \sigma_{Y}}=\frac{E(X Y)-E(X) E(Y)}{\sqrt{E\left(X^{2}\right)-E^{2}(X)} \sqrt{E\left(Y^{2}\right)-E^{2}(Y)}}
$$

Where $X$ is the distance histogram of sample character, $\mathrm{Y}$ is the distance histogram of unknown character histogram. If the correlation $\rho>T_{d}$, then the result is output. If the all similarity coefficient are less than the threshold value of $\mathrm{Td}$, the character will be recognized based on the angle histogram.

The distribution of character pixels in each angle scope is called angle histogram. The standardized character image is divided into $\mathrm{m}$ parts on average. The angle histogram can be drawn in according to the number of character pixels in each angle scope. The standardized angle histogram can be obtained by adjusting the total number of angle histogram bin to 1 .

In the traditional rotated character recognition method, the histogram is counted in different direction and then the histogram is compared with the standard sample character to output the recognition result. This method must count the number of character pixels when the direction is changed, so it will destroy the real-time future of character recognition in embedded device inevitably. Using the character recognition method based on angle histogram, statistics of character is centered on the gravity center of character. So it is a rotational invariance method. When the standardized character is compared with sample character, the result would be output without having to statistical distribution histogram again, as long as the angle histogram is shifted. So the character recognition time can shorten effectively.

The similarity is be calculated by the Equation 5 to compare the rotated character with the sample character. If $\rho>T_{d}$, the recognition result is output. If the all similarity coefficient are less than threshold $T_{d}$, then the recognition entered the stage of comprehensive recognition. 


\section{Experimental Results}

In this section, we present the experimental results of aforementioned character recognition method on simulation data. The experiments were implemented by Matlab R2010a on a computer with Intel Celeron III processor 1.0 GHz CPU, and 512M memory capacity. Recognized target are ten numbers and 52 English letters whose font is Times New Roman. Using the affine transformation matrix $T=\left(\begin{array}{lll}a & b & c \\ d & e & f \\ g & h & 1\end{array}\right)$, the 62 characters are transformed.

Where $L(\beta)$ is scale transformation matrix, the parameter $\beta$ is the scaling factor. $R(\theta)$ is rotation transformation matrix, the parameter $\theta$ is rotation angle. $S(\varphi)$ is shear transformation matrix, $\varphi$ is the shear angle in $x$ direction. The parameters are set respectively as Table 1, and the 62 English characters are transformed by affine matrix. Parts of the transformation results are shown in Fig. 2.

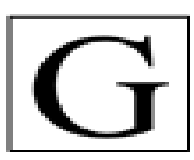

Num: $1 \beta=1, \theta=0$ $\varphi=0$

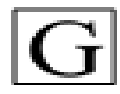

Num:3 $\beta=0.5, \theta=0$ $\varphi=0$

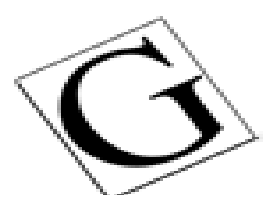

Num:3 $\beta=0.5, \theta=30^{\circ}$ $\varphi=0$

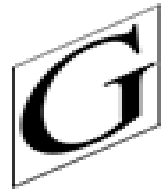

Num:3 $\beta=1, \theta=0^{\circ}$ $\varphi=30^{\circ}$

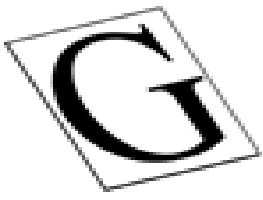

Num:3 $\beta=1, \theta=0^{\circ}$ $\varphi=-15^{\circ}$

Figure 2. simulation data

Table 1 Experimental parameter

\begin{tabular}{cccc|cccc}
\hline Num. & $\beta$ & $\theta$ & $\varphi$ & Num & $\beta$ & $\theta$ & $\varphi$ \\
\hline 1 & 0.5 & 0 & 0 & 11 & 0 & 0 & 5 \\
2 & 0.75 & 0 & 0 & 12 & 0 & 0 & 10 \\
3 & 1 & 0 & 0 & 13 & 0 & 0 & 15 \\
4 & 1.5 & 0 & 0 & 14 & 0 & 0 & 20 \\
5 & 2 & 0 & 0 & 15 & 0 & 0 & 30 \\
6 & 0 & 10 & 0 & 16 & 0.5 & 10 & -5 \\
7 & 0 & 20 & 0 & 17 & 0.75 & 20 & -10 \\
8 & 0 & 30 & 0 & 18 & 1 & 30 & -15 \\
9 & 0 & 40 & 0 & 19 & 1.5 & 40 & -20 \\
10 & 0 & 50 & 0 & 20 & 2 & 50 & -30 \\
\hline
\end{tabular}

Table 2 Experimental result

\begin{tabular}{lccccccccccc}
\hline & $\mathrm{A}$ & $\mathrm{B}$ & $\mathrm{C}$ & $\mathrm{D}$ & $\mathrm{O}$ & $\mathrm{L}$ & $\mathrm{X}$ & $\mathrm{Y}$ & $\mathrm{Z}$ & $\mathrm{a}$ & $\mathrm{b}$ \\
\hline $\begin{array}{c}\text { recognition rate } \\
(\%)\end{array}$ & 100 & 98.4 & 99.5 & 99.4 & 98.3 & 100 & 99.8 & 99.1 & 97.4 & 97.1 & 98.5 \\
$\begin{array}{c}\text { recognition time } \\
(\mathrm{ms})\end{array}$ & 82 & 165 & 85 & 173 & 178 & 67 & 78 & 178 & 167 & 88 & 185 \\
$\begin{array}{c}\text { recognition time } \\
\text { traditional } \\
\text { method(ms) }\end{array}$ & 87 & 581 & 89 & 572 & 588 & 74 & 72 & 590 & 562 & 83 & 620 \\
\hline & $\mathrm{c}$ & $\mathrm{o}$ & 1 & $\mathrm{x}$ & $\mathrm{y}$ & $\mathrm{z}$ & 0 & 1 & 2 & 8 & 9 \\
\hline $\begin{array}{c}\text { recognition rate } \\
(\%) \quad 99.1\end{array}$ & 98.7 & 99.1 & 99.7 & 98.5 & 97.6 & 70.8 & 50.1 & 100 & 100 & 99.5 \\
$\begin{array}{c}\text { recognition time } \\
(\mathrm{ms})\end{array}$ & 173 & 88 & 86 & 190 & 82 & 81 & 190 & 186 & 88 & 81 & 83 \\
$\begin{array}{c}\text { recognition time } \\
\text { traditional } \\
\text { method(ms) }\end{array}$ & 573 & 85 & 82 & 587 & 80 & 84 & 558 & 567 & 90 & 91 & 80 \\
\hline
\end{tabular}

Based on geometry correction character recognition method, the deformed character is recognized. The correct recognition rate and recognition time are shown in Table 2. Due to the limitation of space, 
the part recognition results are listed in the Table 2.As shown in Table 2, the average correct recognition rate reached $97.4 \%$, the highest recognition rate is $100 \%$, and the lowest recognition rate is $50.1 \%$. For all recognition results of 62 characters, the correct recognition rate is more than $90 \%$. At the same time, because of the similarity of $o$ and 0,1 and 1 , correct recognition rate of these characters is less than $90 \%$.

On the other hand, recognition time of aforementioned method is mainly divided into four parts: the time of deformed character standardization; recognition time based on distance histogram; recognition time based on the angle histogram and the comprehensive recognition time. The experimental results show that the average time in four parts: $30 \mathrm{~ms}, 50 \mathrm{~ms}, 90 \mathrm{~ms}$, and $10 \mathrm{~ms}$ respectively. Therefore, the shortest time is about $80 \mathrm{~ms}$, the longest time is about $180 \mathrm{~ms}$ using geometry correction method. Compared with the traditional recognition method, the recognition time is shorted obviously.

\section{Conclusion}

In this paper, a high speed character recognition method for rotated character is presented based on geometry correction. The experimental results of simulation data show that the correct recognition of this method is more than $95 \%$ for $90 \%$ of all English characters and numbers. Because of the similarity of 0 and $\mathrm{O}, 1$ and 1 , the correct recognition rate reduced. But in some applications such as electronic dictionary, the English word and number are not recognized at the same time, so this method is valid for these applications.

\section{Acknowledgements}

This work was supported by the Research Foundation of Education Bureau of Jilin Province under the Grant Number: 2015254.

\section{References}

[1] H. Huang and F. Da: A dictionary learning and KPCA-based feature extraction method for off-line handwritten Tibetan character recognition, Optik - International Journal for Light and Electron Optics, Vol.126 (2015) No.23, p.3795 - 3800.

[2] C. SHI,C. WANG and B. XIAO: Multi-scale Graph-matching Based Kernel for Character Recognition from Natural Scenes, Acta Automatica Sinica, Vol.40 (2014) No.4, p.751 - 756.

[3] M. Ryan and N. Hanafiah: An Examination of Character Recognition on $\backslash\{$ ID $\backslash\}$ card using Template Matching Approach, Procedia Computer Science, Vol.59 (2015) No."", p.520 - 529.

[4] C. Liu and K. Marukawa: Pseudo two-dimensional shape normalization methods for handwritten Chinese character recognition, Pattern Recognition, Vol.38 (2005) No.12, p.2242 - 2255.

[5] N. Narappanawar,B. M. Rao and M. Joshi: Graph theory based segmentation of traced boundary into open and closed sub-sections, Computer Vision and Image Understanding, Vol.115 (2011) No.11, p.1552 - 1558.

[6] J. I. Olszewska: Active contour based optical character recognition for automated scene understanding, Neurocomputing, Vol.161 (2015) No."", p.65 - 71.

[7] A. Sedighi and M. Vafadust: A new and robust method for character segmentation and recognition in license plate images, Expert Systems with Applications, Vol.38 (2011) No.11, p.13497 13504.

[8] M. G. Mora and M. Morini: Local calibrations for minimizers of the Mumford-Shah functional with a regular discontinuity set, Annales de l'Institut Henri Poincare (C) Non Linear Analysis, Vol.18 (2001) No.4, p.403 - 436. 
[9] P. M. Patil and T. R. Sontakke: Rotation, scale and translation invariant handwritten Devanagari numeral character recognition using general fuzzy neural network, Pattern Recognition, Vol.40 (2007) No.7, p.2110 - 2117.

[10] M. S. Chaturvedi and A. A. Kurshid: Implementation for Improved Character Recognition and Classification using SNN Model, Procedia Computer Science, Vol.62 (2015) No."", p.151 - 158. 